Please cite this publication as follow:

Baudena, M., Boni, G., Ferraris, L., von Hardenberg, J. and Provenzale, A.: Vegetation response to rainfall intermittency in drylands: Results from a simple ecohydrological box model, Advances in Water Resources, 30, 1320-1328, doi:10.1016/j.advwatres.2006.11.006, 2007. 


\title{
Vegetation response to rainfall intermittency in drylands: results from a simple ecohydrological box model
}

\author{
M. Baudena ${ }^{\text {a,* }}$, G. Boni ${ }^{a}$, L. Ferraris ${ }^{a}$, J. von Hardenberg ${ }^{\text {b,a }}$ \\ and A. Provenzale ${ }^{b, a}$ \\ ${ }^{a}$ CIMA, Centro di Ricerca Interuniversitario in Monitoraggio Ambientale, Via \\ Cadorna 7, 17100, Savona, Italy \\ ${ }^{\mathrm{b}}$ ISAC-CNR, Istituto di Scienze dell'Atmosfera e del Clima, Corso Fiume 4, \\ 10133 Torino, Italy
}

\begin{abstract}
Vegetation in arid and semi-arid regions is affected by intermittent water availability. We discuss a simple stochastic model describing the coupled dynamics of soil moisture and vegetation, and study the effects of rainfall intermittency. Soil moisture dynamics is described by a ecohydrological box model, while vegetation is represented by site occupancy dynamics in a spatially-implicit model. We show that temporal rainfall intermittency allows for vegetation persistence at low values of annual rainfall volume, where it would go extinct if rainfall were constant. Rainfall intermittency also generates long-term fluctuations in vegetation cover, even in the absence of significant inter-annual variations in the statistical properties of precipitation.
\end{abstract}

Key words: ecohydrology, rainfall intermittency, drylands, mathematical models

\section{Introduction}

Vegetation is part of a complex system where the atmosphere, the living organisms and the soil closely interact on several different spatial and temporal scales. In particular, vegetation dynamics is affected by rainfall and soil properties, and it simultaneously exerts an important control on the water balance [1].

* Corresponding author. 
In drylands, soil moisture is the central control variable, because it summarizes most of the different limiting factors acting in ecosystems characterized by scarce, sporadic and intermittent rainfall [2]. Besides being directly influenced by hydrological, biological and atmospheric processes, soil moisture availability controls processes such as transpiration, primary production and nutrient absorption. In past studies, soil moisture has been used as the only variable in many ecohydrological models, implicitly parameterizing vegetation dynamics [3]. Other works have addressed the coupled dynamics of vegetation and soil moisture, either using an implicit-space approach [4-7], or focussing on pattern formation in spatially-explicit models of vegetation dynamics $[8,9]$.

In this work we explore the role of vegetation feedbacks on evapotranspiration and the effects of temporal rainfall intermittency, a distinguishing feature of arid and semi-arid ecosystems [7]. Soil moisture dynamics is described by a standard ecohydrological box model $[1,3]$ and vegetation dynamics is described by an implicit-space model for site occupancy $[10,11]$.

The results of the analysis indicate that in water-limited ecosystems, temporal rainfall intermittency favours vegetation persistence compared to the case of a constant rain rate. This conclusion agrees with results from experimental investigations, showing that plants from desert ecosystems are favoured in the case of less frequent rainfall events, for the same value of total seasonal rainfall volume $[12,13]$. A minimal amount of water is in fact needed to activate biological processes such as reproduction and seed germination. If precipitation is concentrated in time, this minimum amount of soil water is intermittently exceeded and vegetation cover can persist. In case rainfall is uniformly spread, soil moisture never exceeds the threshold needed to activate plant reproduction and germination and the vegetation cover tends to disappear. This behavior has interesting similarities with the effects of spatial rainfall intermittency [2] and/or of inhomogeneous spatial redistribution of rainfall by runoff and differential infiltration $[8,9]$. In all these cases, the spatially or temporally inhomogeneous water availability allows soil moisture to exceed, in some places and at some times, the minimum threshold for plant survival that would not be reached if water were distributed homogeneously. 


\section{Ecohydrological models}

\subsection{Probabilistic model for soil moisture dynamics}

We describe the global water balance in the upper soil layer by the model of Laio et al [14], also discussed in [1,3]:

$$
\frac{\mathrm{d} s}{\mathrm{~d} t}=\frac{1}{n Z_{r}}[\varphi(s, r)-\chi(s)]=I(s, r)-X(s)
$$

where $s$ is relative soil moisture averaged over the root zone $(0 \leq s \leq 1), n$ is soil porosity and $Z_{r}$ is the active soil depth in millimeters. The quantity $r$ is daily rainfall, $\varphi(s, r)$ is the rainfall infiltration rate and $\chi(s)$ represents soil moisture losses from evapotranspiration and leakage, all in units of millimeters of water per day $\left(\mathrm{mm} \mathrm{day}^{-1}\right)$. These terms are normalised as $I=\varphi / n Z_{r}$ and $X=\chi / n Z_{r}$, which have units of day $^{-1}$.

Equation (1) represents soil moisture dynamics at daily time scale; soil moisture diffusion is assumed to be negligible. The model does not resolve the vertical structure of the soil layer and the propagation of the wetting front. In addition, the description formulated in eq. (1) does not take into account spatial variations of soil moisture and thus represents a box-model for the average soil moisture in a given region, assuming statistical homogeneity of rainfall in the study area and rapid water redistribution by surface runoff. For a different approach, that explicitely resolves the spatial structure of the soil moisture field, see e.g. [9].

The infiltration rate is assumed to be equal to the rainfall rate, as long as the soil layer is not saturated; when rainfall exceeds the available water storage in the soil, the excess is converted into surface runoff. Thus,

$$
I=\left\{\begin{array}{lll}
\frac{r}{n Z_{r}} & \text { if } & \frac{r \Delta t}{n Z_{r}}<1-s \\
\frac{1-s}{\Delta t} & \text { if } & \frac{r \Delta t}{n Z_{r}} \geq 1-s
\end{array}\right.
$$

where $\Delta t=1$ day.

Since we focus on arid and semi-arid regions, we assume that rainfall is present only during the wet season, which has a duration of $N_{\text {wet }}$ days. During the wet season, precipitation is either kept constant at the daily rainfall rate $\bar{r}$, or it is intermittently distributed. In the intermittent case, rainfall occurrence is represented as a sequence of discrete events, assumed to be concentrated at an instant in time. This assumption is consistent with the fact that rainfall 
events in water-limited ecosystems are usually of short duration (a few hours at most), and are thus assumed to be instantaneous at the daily time scale adopted here.

For intermittent rainfall, the occurrence of the events is simulated with a Poisson process with expected interarrival time $N$. Each occurrence is associated with a rainfall depth that is exponentially distributed with expected value $r_{0}$ [1]. Since the two processes determining the rainfall volume for each event and the interarrival times are independent, the average daily rainfall during the wet season becomes $\bar{r}=r_{0} \bar{N}_{e v} / N_{\text {wet }}$ where $\bar{N}_{e v}$ is the average number of rain events in a season. Average annual rainfall is obtained as $\overline{R_{y}}=\bar{r} N_{w e t} \Delta t=r_{0} \bar{N}_{e v} \Delta t$. In the case of intermittent rainfall, infiltration becomes a stochastic, statedependent input that takes the form of multiplicative noise, since it modulates the external random forcing due to rainfall by the value of soil moisture.

In the following, we shall make a distinction between water losses from vegetated soil, which we call $X_{b}(s)$, and losses from bare soil, which we call $X_{0}(s)$. In vegetated soil, water losses are the sum of direct evaporation from the soil plus plant transpiration, $E(s)$, and leakage, $L(s)$, i.e. $X_{b}(s)=E(s)+L(s)$, see Fig. 1. When the soil moisture content is high enough for the plants to be fully functional (with open stomata), the total evapotranspiration $E(s)$ depends mainly on the type of plant and on climatic conditions. In a given environment, we assume that plant transpiration in optimal conditions occurs at a constant rate $E_{\max }$ [1]. When soil moisture falls below a critical value, $s^{*}$, plants start reducing transpiration by closing their stomata. Below a lower critical value $s_{w}$, called the wilting point, plants fully close their stomata and start to wilt. $E(s)$ is assumed to decrease linearly with $s$ between $s^{*}$ and $s_{w}$. Below $s_{w}$, only evaporation contributes to soil water depletion: Evaporation decreases from $E_{w}$ at $s_{w}$ to zero at the hygroscopic point, $s_{h}$, see Fig. 1.

Leakage losses are due to the gravitational pull on soil water, and they are especially important when the soil moisture exceeds the soil field capacity, $s_{f c}$. Here we represent leakage losses by an exponential growth starting at the soil field capacity $s_{f c}$ and reaching the saturated hydraulic conductivity $K_{s}$ at $s=1$, see Fig. 1 .

The full expression for the water losses from vegetated soil thus becomes

$$
X_{b}= \begin{cases}0 & \text { if } s \leq s_{h} \\ E_{w} \frac{s-s_{h}}{s_{w}-s_{h}} & \text { if } s_{h}<s \leq s_{w} \\ E_{w}+\left(E_{\max }-E_{w}\right) \frac{s-s_{w}}{s^{*}-s_{w}} & \text { if } s_{w}<s \leq s^{*} \\ E_{\max } & \text { if } s^{*}<s \leq s_{f c} \\ E_{\max }+K_{s} \frac{e^{\beta\left(s-s_{f c}\right)}-1}{e^{\beta\left(1-s_{f c}\right)}-1} & \text { if } s_{f c}<s \leq 1\end{cases}
$$




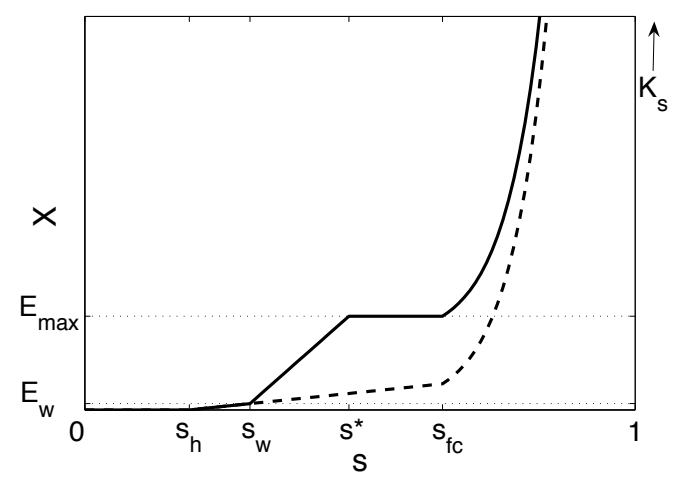

Fig. 1. Total water losses for vegetated soil, $X_{b}$ (continuous line), and for bare soil, $X_{0}(s)$ (dashed line), as a function of relative soil moisture, $s(0 \leq s \leq 1)$. The value $s_{h}$ is the hygroscopic point below which no evaporation takes place, $s_{w}$ is the wilting point below which plants start to wilt, $s^{*}$ is the critical soil moisture value below which plants start reducing transpiration by closing their stomata, and $s_{f c}$ is the soil field capacity above which leakage occurs. The value $E_{w}$ defines the value of pure evaporation at $s_{w}$ and $E_{\max }$ is the optimal evapotranspiration value for $s \geq s^{*}$. The (large) value $K_{s}$ is the saturated hydraulic conductivity at $s=1$ and it measures the maximum intensity of leakage.

Table 1

List of parameters related to soil characteristics, used in the model for three different soil textures. ${ }^{\mathrm{a}}$

\begin{tabular}{lccccccc}
\hline & $n$ & $K_{s}(\mathrm{~mm} / \mathrm{d})$ & $\beta$ & $s_{h}$ & $s_{w}$ & $s^{*}$ & $s_{f c}$ \\
\hline Loam & 0.45 & 200 & 14.8 & 0.19 & 0.24 & 0.57 & 0.65 \\
Loamy sand & 0.42 & 1000 & 12.7 & 0.08 & 0.11 & 0.31 & 0.52 \\
Sand & 0.35 & 2000 & 12.1 & 0.08 & 0.11 & 0.33 & 0.35 \\
\hline
\end{tabular}

a Other parameters used: $E_{\max }=4.5 \mathrm{~mm} / \mathrm{d}, E_{w}=0.1 \mathrm{~mm} / \mathrm{d}, Z_{r}=300 \mathrm{~mm}$, $r_{0}=15 \mathrm{~mm} / \mathrm{d}, N_{\text {wet }}=200 \mathrm{~d}$.

The parameter values used in the model depend on the soil and vegetation types. In the following, we adopt the parameter values listed by RodriguezIturbe and Porporato [1], which are reported in Table 1, typically corresponding to herbaceous vegetation.

In bare soil, water losses have a simpler expression as there is no plant transpiration. In this case, we assume water losses to be described by $X_{0}(s)=$ $E_{0}(s)+L(s)$, where $E_{0}(s)$ is pure evaporation from the soil. Leakage $L(s)$ keeps the same expression as for vegetated soil, while we assume that $E_{0}$ vanishes below the hygroscopic point $s_{h}$ and then increases linearly up to field capacity $s_{f c}$, with the requirement that $E_{0}\left(s_{w}\right)=E_{w}$. Thus, $X_{0}$ and $X_{s}$ coincide below the wilting point $s_{w}$, while $X_{b}>X_{0}$ above $s_{w}$. The full expression 
for water losses from bare soil becomes

$$
X_{0}= \begin{cases}0 & \text { if } s \leq s_{h} \\ E_{w} \frac{s-s_{h}}{s_{w}-s_{h}} & \text { if } \quad s_{h}<s \leq s_{f c} \\ E_{w} \frac{s_{f c}-s_{h}}{s_{w}-s_{h}}+K_{s} \frac{e^{\beta\left(s-s_{f c}\right)}-1}{e^{\beta\left(1-s_{f c}\right)}-1} & \text { if } \quad s_{f c}<s \leq 1\end{cases}
$$

At field capacity, evaporation from bare soil is $E_{w}\left(s_{f c}-s_{h}\right) /\left(s_{w}-s_{h}\right)$. Depending upon the type of soil, this is about ten times larger than $E_{w}$, i.e., about $1 \mathrm{~mm} /$ day, but definitely smaller than the optimal evapotranspiration from vegetated soil, $E_{\max }$.

The treatment of evaporation from bare soil adopted in this model is clearly simplified. In arid and semi-arid regions, only the first 5 to $10 \mathrm{~cm}$ of bare soil dry out due to evaporation, whereas the underlying soil layers remain wet and function as water storage $[4,15]$. In the upper layer, potential evaporation can be very large, significantly exceeding plant transpiration due to water uptake by roots at deeper layers. In our modelling exercise, we consider a soil layer of $30 \mathrm{~cm}$, significantly deeper than the layer directly affected by evaporation, in line with previous works ([1] and references therein). For this reason, the value of evaporation rate that we use is a depth-weighted average between the large evaporation rate from the upper soil portion and the practically null evaporation rate from the lower soil layer, where only root uptake and leakage play a role. As a result, the evaporation rate in bare soil is less than evapotranspiration from vegetated soil, and the value of $E_{w}$ should thus be interpreted as an average over the whole soil layer depth considered here.

\subsection{Implicit-space description of vegetation dynamics}

Vegetation dynamics is described using an implicit-space logistic equation, originally introduced by Levins [10], and later generalised and applied to vegetation competition by Tilman [11]. In this approach, the soil surface is subdivided into neighbouring parcels (or sites), which may be vegetated or empty. The fraction of vegetated sites is denoted by $b$, where $0 \leq b \leq 1$. Variations in site occupancy depend on the rate at which empty sites are colonized and on the rate at which vegetated sites become vacant owing to mortality. Individual site-by-site processes of death and replacement are averaged over the whole habitat. Based on these assumptions, one can write an evolution equation for the fraction of sites occupied by vegetation as [11]:

$$
\frac{\mathrm{d} b}{\mathrm{~d} t}=g b(1-b)-\mu b
$$


where $g$ is the colonization rate (per occupied site) and $\mu$ is the local extinction rate, i.e. the local vegetation mortality. The propagule production rate, $g b$, is multiplied by the fraction of empty sites, $(1-b)$, to give the total growth rate of the fraction of vegetated sites due to colonization (a new plant that establishes itself in a previously occupied site does not change the number of occupied sites). In this simplified description, the colonization rate $g$ lumps together the ability of plants to produce seeds and the germination probability once the seeds have reached an empty site. The seeds themselves are assumed to be randomly and uniformly dispersed over the whole area of study (in the case of a spatially-explicit approach, this would correspond to a global coupling between the sites). The probability that an occupied site becomes vacant due to mortality is assumed to be density-independent, and thus the number of occupied sites decreases at the rate $\mu b$.

This equation has two fixed points: $b_{0}=0$, which is stable for $\mu>g$, and $b_{1}=1-\frac{\mu}{g}$, stable for $\mu<g$. When a species has non-zero mortality $(\mu>0)$ and it has finite colonization ability $(g<\infty)$, there is always a portion of space that remains empty. This result indicates that a single species can never occupy the whole available space and it has been used to rationalize the coexistence of different competing species [11].

\section{The coupled dynamics of soil moisture and vegetation}

In this section we compare the behavior of vegetation subject to constant rainfall with that taking place under intermittent conditions. We assume that rainfall is present only during the wet season, which is fixed to have a duration of $N_{w e t}=200$ days (changes in the value of $N_{\text {wet }}$ do not qualitatively affect the results).

During the dry season, we consider two opposite, and extreme, situations. The first option is that both vegetation and soil moisture dynamics are "frozen", so that soil moisture and biomass remain constant during the dry season. This is motivated by dryland conditions where soil moisture below the upper 10 $\mathrm{cm}$ of soil is protected from evaporation and vegetation slows down or almost ceases physiological activities during the dry season. The second option is that vegetation and soil moisture dynamics continue unchanged, but without rainfall input, during the dry season, leading to rapid soil drying and severe vegetation stress. Real ecosystems behave in intermediate ways [2]; considering the two extremal strategies helps bracketing the range of possible behaviors. We shall return to this point later.

To include the dependence of biomass dynamics on soil moisture, we assume 


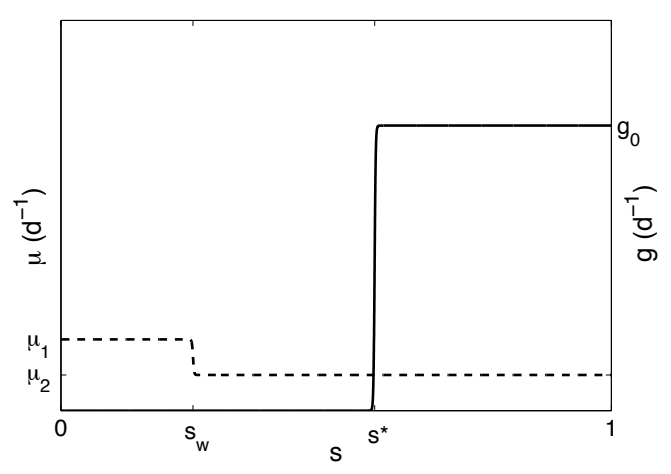

Fig. 2. Colonization rate $g$ (solid line) and local extinction rate $\mu$ (dashed line) as a function of soil moisture $s$. The value $s_{w}$ is the wilting point and $s^{*}$ is the critical soil moisture value below which plants start reducing transpiration by closing their stomata. The value $g_{0}$ is the optimal colonization rate for $s \gg s^{*}, \mu_{1}$ is the local mortality rate for dry soil, $s \ll s_{w}$, and $\mu_{2}<\mu_{1}$ is the local mortality rate for moist soil, $s \gg s_{w}$.

that the colonization and extinction rates depend on $s$ :

$$
\frac{\mathrm{d} b}{\mathrm{~d} t}=g(s) b(1-b)-\mu(s) b
$$

As before, the variable $b$ represents the fraction of land surface occupied by vegetation and $s$ is the average relative soil humidity in the study area.

In the following, we assume the colonization rate $g$ to be close to zero when soil moisture is significantly below the fully-open-stomata threshold $s^{*}$. When $s>$ $s^{*}$, we assume that $g(s)$ approaches a constant value $g_{0}$. With these choices, we intend to crudely model the fact that vegetation has poor colonization ability below $s^{*}$, both because the existing vegetation suffers from water stress and it can allocate fewer resources to seed production, and because seeds can have lower germination probability when the soil is not sufficiently moist. In practice, we model $g(s)$ with a steep hyperbolic tangent centered around $s^{*}$, $g(s)=\frac{g_{0}}{2}\left[1+\tanh \frac{s-s^{*}}{a}\right]$ with $a=0.002$ (see Fig. 2).

The extinction rate $\mu$ is assumed to depend on soil moisture, and it is maximal under wilting conditions. In our model, $\mu$ tends to the constant value $\mu_{1}$ for $s<s_{w}$ and to the value $\mu_{2}$ for $s>s_{w}$, where $\mu_{1}>\mu_{2}$. Again, we assume a hyperbolic tangent shape centered around $s_{w}, \mu(s)=\frac{\mu_{1}+\mu_{2}}{2}+\frac{\mu_{1}-\mu_{2}}{2} \tanh \frac{s_{w}-s}{a}$ with $a=0.002$, see Fig. 2 .

To close the system, we need to take into account the effects of the presence or absence of vegetation on soil moisture. To do so, we make a distinction between evapotranspiration from vegetated sites and evaporation from bare sites, and we use the two loss functions introduced above, $X_{b}$ and $X_{0}$, for vegetated and bare sites respectively. The total losses are obtained by weighting $X_{b}$ and $X_{0}$ 
respectively by the fraction of vegetated soil, $b$, and of bare soil, $1-b$. The coupled soil-vegetation system becomes:

$$
\left\{\begin{array}{l}
\frac{\mathrm{d} s}{\mathrm{~d} t}=I(s, r)-\left[b X_{b}(s)+(1-b) X_{0}(s)\right] \\
\frac{\mathrm{d} b}{\mathrm{~d} t}=g(s) b(1-b)-\mu(s) b
\end{array}\right.
$$

In the following discussion, we first focus on the case with frozen dry-season dynamics. When rainfall is kept constant during the wet season, the system has multiple fixed points whose value and stability depend on the annual rainfall. Below a critical rainfall height, $b=0$ is the only stable fixed point. The bare state becomes unstable above this critical rainfall value, where another stable fixed point with $b \neq 0$ appears through a transcritical bifurcation. The fraction of vegetated surface grows with the average rainfall until it reaches the carrying capacity of the system, $1-\frac{\mu}{g_{0}}$, where $g_{0}$ is the maximum value of $g$.

When temporal rainfall intermittency is introduced, vegetation cover and soil moisture do not tend to constant values but they fluctuate around average values $\bar{b}$ and $\bar{s}$. Figure 3 shows example series of vegetation cover and soil moisture as a function of time. The constant intervals correspond to the frozen states during the dry seasons.

To explore the effects of rainfall intermittency, in figure 4 we compare the fixed points of vegetation cover and soil moisture obtained for constant rain during the wet season with the average values obtained for intermittent precipitation, as a function of the total annual rainfall volume. The curves in the three panels show vegetation cover versus rainfall (a), relative soil moisture versus rainfall (b), and vegetation cover versus soil moisture (c), for three soil types and for constant rainfall. The symbols in the three panels of Figure 4 show the average values of vegetation cover (a) and soil moisture (b) as a function of average annual rainfall, and the average vegetation cover versus the average soil moisture (c), for the case of intermittent rainfall. For an easy comparison with real conditions, rainfall is expressed by its annual value, $\overline{R_{y}}=\bar{r} N_{\text {wet }} \Delta t$.

The average fraction of space covered by vegetation, $\bar{b}$, display an interesting behavior as a function of rainfall: $\bar{b}$ is nonzero also for precipitation values that lead to zero vegetation coverage in the case of constant rainfall, and it remains larger than the constant-rain value up to about a total annual rainfall of 700 $\mathrm{mm}$. Therefore, precipitation intermittency allows for vegetation persistence at lower levels of water input than those needed for constant rainfall. On the other hand, the average soil moisture for intermittent rainfall is generally lower than for constant rain, due to the fact that the larger fraction of vegetated sites leads to larger water losses as evapotranspiration from vegetated sites is larger than pure evaporation from bare soil. 
(a)

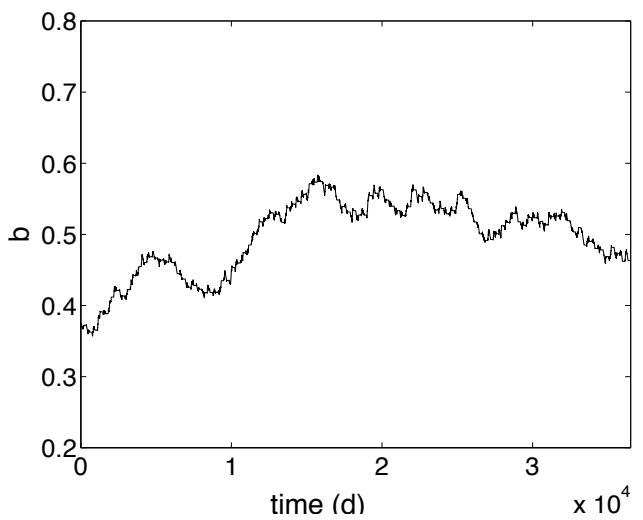

(b)

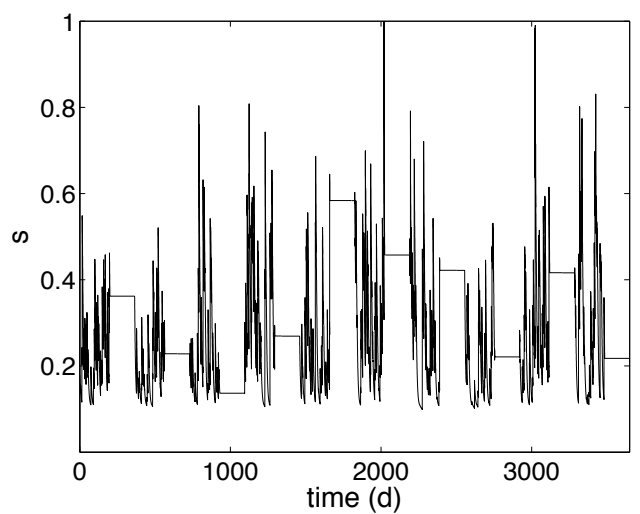

Fig. 3. Time series of vegetation cover (a) and soil moisture (b). The average rainfall intensity of each event is $r_{0}=15 \mathrm{~mm}$ day $^{-1}$ and the mean interarrival time between rainfall events is $N=5$ days, which gives average annual rainfall $\overline{R_{y}} \approx 550 \mathrm{~mm}$. The duration of the wet season is set at 200 days [1] and the dry-season dynamics is frozen. The parameter values of the ecohydrological model are reported in Table 1. The vegetation parameters are $g_{0}=0.8 \mathrm{y}^{-1}, \mu_{1}=0.2 \mathrm{y}^{-1}, \mu_{2}=0.1 \mathrm{y}^{-1}[11,6]$. Note the different time scales in the two panels.

In figure 4 , we used a maximal colonization rate $g_{0}=0.8 \mathrm{y}^{-1}$. In the case of "frozen" dry season dynamics, the results are rather insensitive to (realistic) changes in colonization rate and vegetation mortality.

We next consider the other case of an "active" dry season, during which soil moisture and biomass react dynamically but the rainfall input is zero. In real ecosystems, plants adopt different strategies to exploit water resources under soil-drying conditions [2]. As mentioned above, the two configurations considered above and in the following can be seen as two extreme types of behavior.

When rainfall is kept constant during the wet season, and set to zero in the dry season, vegetation and soil moisture oscillate between two values, relaxing to the two fixed points corresponding respectively to rainy and dry conditions. 
(a)

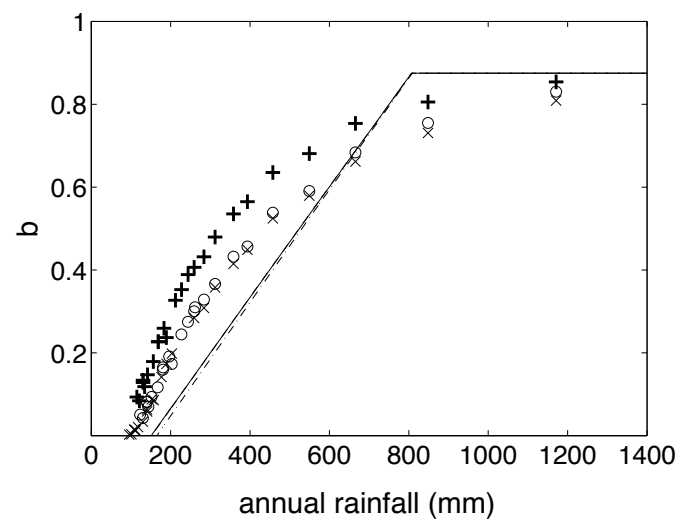

(b)

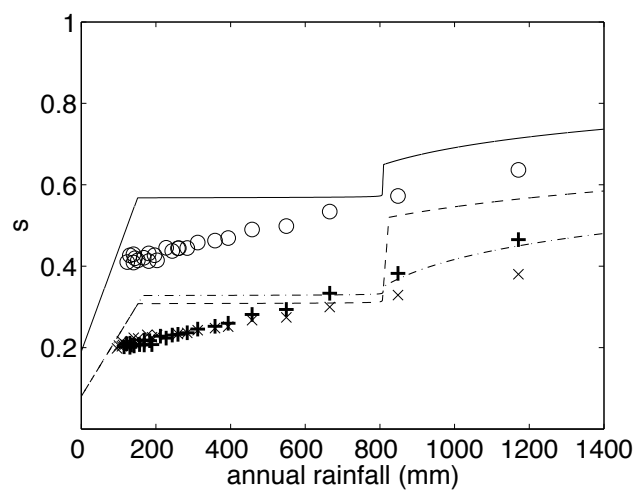

(c)

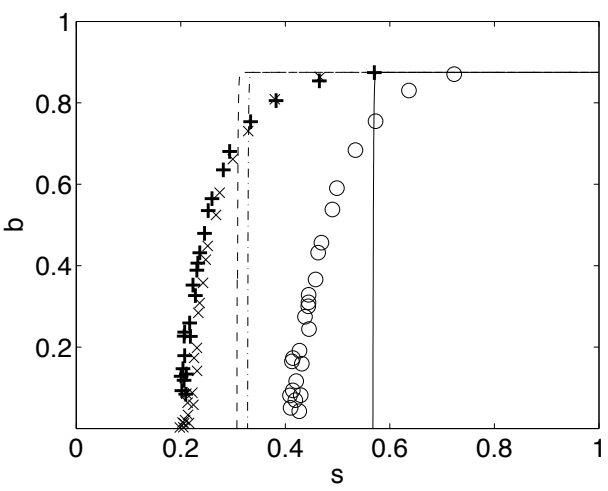

Fig. 4. Comparison of the cases with constant and intermittent precipitation. In each panel, the lines represent stable fixed points of the system for three different kind of soils (solid line, loam; dashed line, loamy sand; dashdot, sand), whereas the markers stand for average values in the case of intermittent precipitation (circles for loam, + marks for loamy sand and x-marks for sand). (a) (Average) vegetation cover as a function of (average) annual rainfall. The stable fixed points corresponding to different soil types are almost coincident. (b) (Average) soil moisture as a function of (average) annual rainfall. (c) (Average) vegetation cover versus (average) soil moisture. The parameter values of the ecohydrological model are reported in Table 1. In panels (a) and (b), rainfall is expressed by its annual value, $\overline{R_{y}}=\bar{r} N_{\text {wet }} \Delta t$; the total annual rainfall is varied by changing the average interarrival time [16]. The duration of the wet season is set at 200 days [1]. The vegetation parameters are $g_{0}=0.8 \mathrm{y}^{-1}, \mu_{1}=0.2 \mathrm{y}^{-1}, \mu_{2}=0.1 \mathrm{y}^{-1}[11,6]$. 
(a)

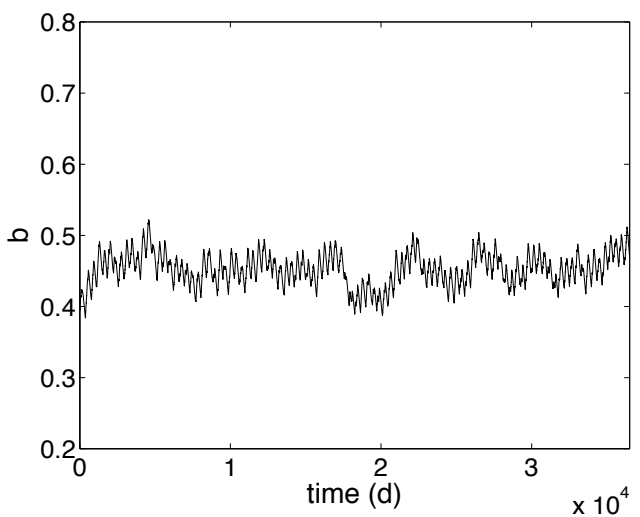

(b)

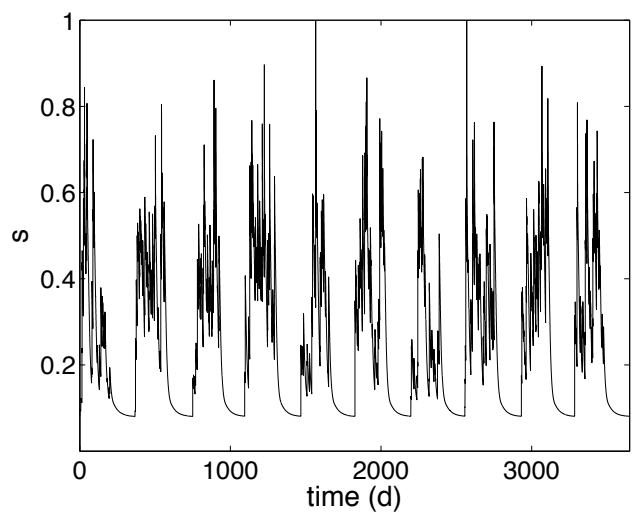

Fig. 5. Time series of vegetation cover (a) and soil moisture (b), for the same parameter values used in Figure 3. The average rainfall intensity of each event is $r_{0}=15 \mathrm{~mm} \mathrm{day}^{-1}$ and the mean interarrival time between rainfall events is $N=5$ days, which gives the average annual rainfall $\overline{R_{y}} \approx 550 \mathrm{~mm}$. Vegetation and soil moisture dynamics are assumed to continue unchanged during the dry season with zero precipitation input. Note the different time scales in the two panels.

The vegetation cover is smaller than for the case where vegetation dynamics is frozen during the dry season. However, the threshold between vegetation presence and bare soil does not change significantly.

When rainfall intermittency is introduced, in the wet season vegetation and soil moisture undergo significant fluctuations, and relax towards zero during the dry season. Figure 5 shows two example time series of vegetation cover and soil moisture, in the case with vegetation feedback on evapotranspiration and active dynamics during the dry season.

In the case of active dry season, vegetation response to rainfall intermittency varies with plant colonization ability: Intermittency does not give a clear advantage to plants with lower colonization rates, shown in fig. 6a, with a slight exception for loamy sand where also plants with low colonization rate are favoured by rainfall intermittency. On the other hand, Figure $6 \mathrm{~b}$ shows that 
(a)

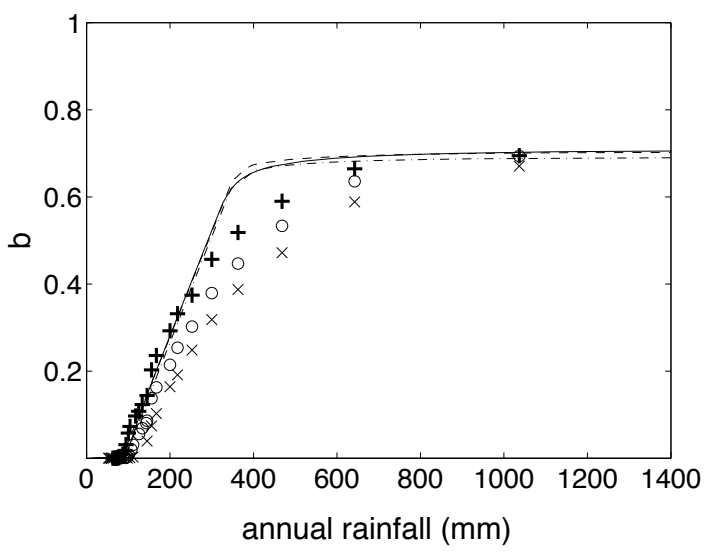

(b)

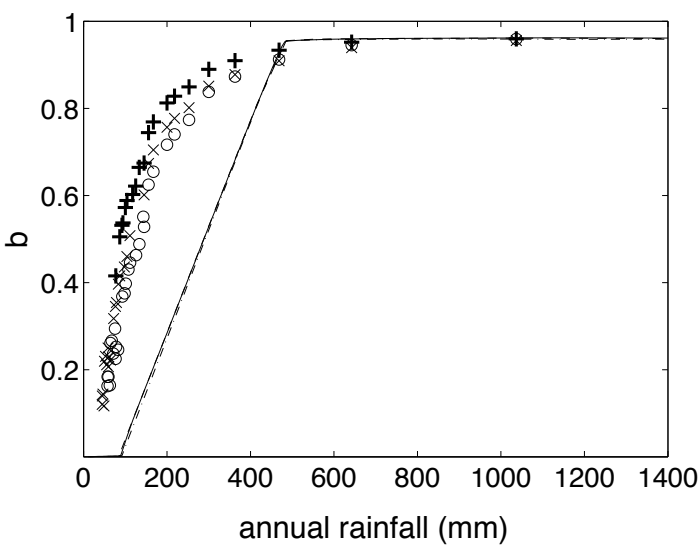

Fig. 6. Average vegetation cover versus annual rainfall, for the system with active dynamics in the dry season. Lines are for constant rainfall during the wet season, markers for intermittent rainfall in the wet season. Rainfall is expressed by its annual value, $\overline{R_{y}}=\bar{r} N_{\text {wet }} \Delta t$. The top panel shows the case of plants with low colonization rate $\left(g_{0}=0.8 \mathrm{y}^{-1}\right)$. The bottom panel shows the case of high colonization rate $\left.\left(g_{0}=8 \mathrm{y}^{-1}\right)\right)$. Vegetation is definitely favoured by rainfall intermittency for high colonization rates, while it is only marginally favoured or disfavoured in the case of low colonization rates. Three different kind of soils are considered (loam, solid line and circles; loamy sand, dashed line and + marks; sand, dashdotted line and $\mathrm{x}$-marks). For parameter values, see table 1 and the caption of fig. 4 .

in case of relatively high colonization rates, vegetation is significantly favoured by temporal rainfall intermittency.

\section{Discussion}

The above results can be rationalized by noticing that in arid conditions vegetation is usually favoured by fluctuations in relative soil moisture, $s$ : Given the shape of $g(s)$, one has that for $\bar{s}<s^{*}, \overline{g(s)} \geq g(\bar{s})$. On the other hand, it 
is also true that for $\bar{s}>s_{w}$ one has $\overline{\mu(s)} \geq \mu(\bar{s})$, and thus the value of $\overline{b(s)}$ depends on a balance between the positive effect coming from a larger value of $\bar{g}$ and the negative effect coming from a possibly larger value of $\bar{\mu}$ if soil moisture goes too often below $s_{w}$. For $\bar{s}$ sufficiently close to $s^{*}$, and moderate soil moisture fluctuations, the results of our analysis indicate that the positive effect is dominant and vegetation persists. For small fluctuations in $s$ close to $s^{*}$, one can consider $\mu(s) \approx \mu_{2}$ and use the standard expansion of a nonlinear, stochastically varying function,

$$
\overline{g(s)}=\overline{g(\bar{s}+\delta s)} \approx g(\bar{s})+\frac{1}{2}\left(\frac{d^{2} g}{d s^{2}}\right)_{s=\bar{s}} \overline{(\delta s)^{2}}
$$

where $\delta s$ is the random fluctuation in soil moisture and we have assumed $\overline{\delta s}=0$. Thus, for $\bar{s}<s^{*}$, where the second derivative of $g(s)$ is positive, $d^{2} g / d s^{2}>0$, and for small fluctuations in $s$ one has $\overline{g(s)}>g(\bar{s})$, as observed. At values $\bar{s}>s^{*}$, the effect of fluctuations becomes negative and in this case $\overline{g(s)} \leq g(\bar{s})$.

The above arguments indicate that soil moisture fluctuations are usually beneficial for $\bar{s}<s^{*}$, where often $\overline{b(s)}>b(\bar{s})$. This can be observed in the plots of vegetation cover as a function of soil moisture (e.g. Fig. 4 (c)). However, this is not necessarily reflected in the plots of vegetation cover versus the average rainfall depth. In this case, precipitation intermittency can become a disadvantage: The relationship between $s$ and $r$ is often less favorable in the intermittent case, where all components of water loss from the system (such as runoff, evapotranspiration and leakage) increase. Thus, the consequences of rainfall intermittency are determined by the balance between the benefits due to soil moisture fluctuations and the negative effect of a lower average soil moisture: larger colonization abilities bring enough advantage to overcome the damage from increased soil water losses. On the other hand, lower colonization abilities do not allow plants to take full advantage of rainfall intermittency. Thus, regions characterized by high rainfall intermittency should be preferentially populated by plants with high colonization rates (i.e., high seed production and/or high probability of seed germination). A special case happens for loamy sand soils, where, on one hand, lower hydraulic conductivity increases soil water availability as compared to coarser soils, and on the other hand, lower values of $s_{h}, s_{w}$ and $s^{*}$ allow for more water to be extracted than from finer soils [14].

The above discussion indicates that the effects of rainfall intermittency depend on the shape of the colonization rate $g$. To have a significant effect, the colonization rate must have a nonlinear dependence on the soil moisture $s$, with positive second derivative. Whenever this is true, the picture does not change qualitatively. However, if the colonization rate has an approximately linear dependence on $s$ (with vanishing second derivative), then the beneficial 


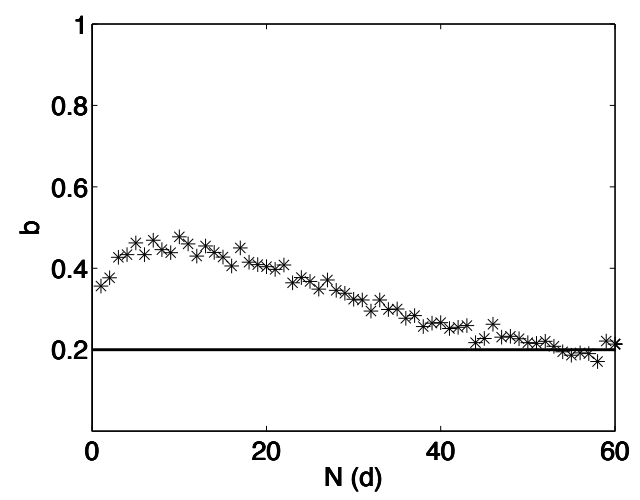

Fig. 7. Average vegetation cover versus the level of rainfall intermittency, as expressed by the value of the interarrival time of rainfall events, $N$. The total annual rainfall is fixed at $300 \mathrm{~mm} /$ year; larger values of $N$ correspond to larger intensity of individual rainfall events. The horizontal line represents vegetation cover in the case of constant rainfall. Same parameters as in figure 4, for loamy sand and frozen dynamics during the dry season.

effect of rainfall intermittency vanishes as well.

Previous works have suggested that biomass response may depend on the level of intermittency, i.e., on the number and intensity of the individual rainfall events [17-19]. We explored this issue by varying the number and intensity of rainfall events for a fixed value of total annual rainfall. Figure 7 shows the case for an annual rainfall of $300 \mathrm{~mm} / \mathrm{y}$ on loamy sand and frozen dynamics during the dry season. The value of $b$ shows a slight growth with the level of intermittency (as measured by the value of the interarrival time $N$ in days), up to an optimal value of rainfall intermittency which in this case corresponds to an average interarrival time of about ten days. At larger values of $N$, the vegetation cover decreases with increasing intermittency, due to the fact that individual events have a large rainfall intensity and a significant portion of rain water is lost by runoff. The effect of intermittency is beneficial as the average vegetation cover is larger than that obtained for constant rainfall.

An interesting point concerns the properties of the temporal fluctuations in vegetation cover for the two cases considered in the analysis. Figure 8 shows the power spectra of vegetation cover for the frozen-dry-season case (a), and the active dry season situation (b), as a function of the frequency $\nu$. In the case of active dry season, a peak at the annual frequency is visible, owing to the yearly oscillation introduced by the decaying vegetation cover during the dry season. Apart from this peak, the two spectra are quite similar, and all display a "red", broad-band appearance characterized by increasing variance for decreasing frequency, similarly to what happens when a climatic component with slow reaction time is stochastically forced by white noise [20]. 
(a)

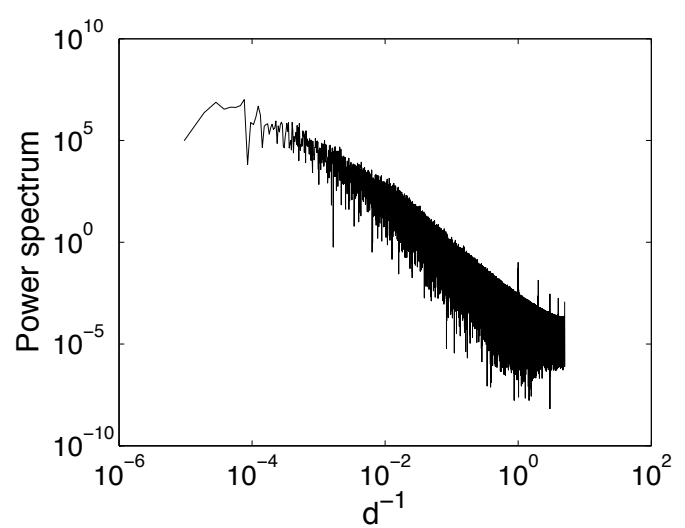

(b)

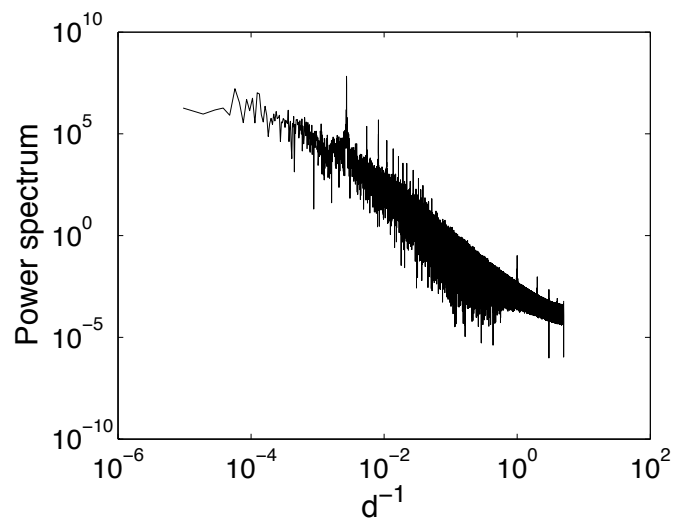

Fig. 8. Power spectral densities of vegetation cover on loamy sand, for interarrival time $N=5$ days (about $550 \mathrm{~mm}$ of rainfall per year). (a) Frozen dry season, and (b) active dynamics during the dry season.

Clearly, in realistic situations the statistical properties of rainfall can display long-term fluctuations that affect ecosystem functioning, as discussed e.g. in [21]. However, our results indicate that long-term fluctuations at decadal scales in vegetation cover should anyway be expected, even in the absence of climatic drifts. Only at the lowest frequencies, corresponding to periodicities longer than about 30-50 years, some saturation in the spectrum starts to emerge. In nature, vegetation cover and ecosystem functioning will display long-term fluctuations due to the interplay of climate variability (the "forcing") and of the internal dynamics of biomass.

The model discussed here is extremely simplified, and it has several aspects that can be further improved. In particular, we only considered the feedback of vegetation on evapotranspiration, and did not include either the reduction of evaporation due to shading or the increase of infiltration as a consequence of vegetation cover $[8,9]$. In the case considered here, plant transpiration greatly exceeds evaporation from the soil and the shading feedback is assumed to have limited impact. A simplified way to include it is by slightly decreasing 
the value of $E_{\max }$; a sensitivity study on the value of the parameters shows that reasonable changes in $E_{\max }$ and/or $E_{w}$ do not qualitatively alter the results.

The presence of an infiltration feedback can be important, but it is hardly represented in a model which considers a single, globally-averaged value for soil moisture due to rapid rainfall redistribution by surface runoff. A future extension of the model will consider the separate dynamics of soil moisture in bare and vegetated soil; in this case, the infiltration feedback can be properly included.

A further limitation of the model is that we considered only the two extreme cases of "active" or "frozen" dynamics during the dry season. These should not be taken as representing realistic situations but rather as extreme types of behavior that bracket the realistic cases. In addition, rainfall and growing season are not always in phase: In cases like South Africa [1] or in the Negev desert this is relatively true, but in Mediterranean climates the situation can be rather different. The model discussed here deals only with situations where rainfall and growing season are in phase, with a specific focus on grasslands and annual plants or shrubs [11].

Finally, the current model does not distinguish between the upper soil layer where both evaporation and transpiration take place and the lower layer where only transpiration due to root uptake is present. Although the use of a depthaveraged evaporation rate allows for reasonably representing the mean evaporation from the soil layer, in extremely arid conditions the simplified form of evaporation adopted here can force the whole 30-cm-deep soil layer to reach the hygroscopic point, while in nature only the upper portion of layer would reach $s_{h}$. Future extensions of the model will include the use of two soil layers to better represent evaporation losses from bare soil; this could be useful to model coexistence of vegetation types with root depth separation (e.g. shrubs and annual grass, see [22]).

\section{Summary and conclusions}

In this paper we discussed a ecohydrological box model for the coupled dynamics of vegetation and soil moisture, focussing on the effect of rainfall intermittency. Although simplified, this model is useful for revealing relevant processes. The results of the analysis show that temporal intermittency of rainfall allows for vegetation persistence at lower annual precipitation rates than in the case of constant rainfall. This behavior is robust to reasonable changes in the values of the system parameters. Although the quantitative details can vary, the overall qualitative picture remains the same. 
Since our focus is on arid and semi-arid regions, we considered the case of seasonal rainfall, assumed to be fully concentrated in a wet season during which rain is either kept constant or intermittently distributed. During the dry season, we considered two extreme choices for the dynamics of vegetation and soil moisture: Either we assumed that the dynamics are frozen, and thus vegetation and soil moisture maintain the values that had at the end of the previous wet season, or we assumed that vegetation and soil moisture dynamics are the same as during the wet season, with no rainfall, full evapotranspiration, and null vegetation colonization rate. The second case is clearly the most demanding for vegetation, which during the dry season is subject to strong water stress due to continued evapotranspiration and no water input. Even in this unfavorable case, the persistence of vegetation cover is still favoured by intermittency, but generally only if plants can colonize new sites fast enough during the wet season.

As a final comment, we note that since rainfall intermittency plays a role in determining vegetation persistence in drylands, climatic changes that lead to variations in the temporal distribution of precipitation, even without affecting the total annual rainfall volume, can have significant impact on vegetation cover. On the other hand, long-term fluctuations in vegetation cover at decadal time scales, as indicated by the spectral densities, are naturally generated also in the case of statistically-stationary rainfall forcing.

We are grateful to Moshe Shachak for discussions and suggestions. We thank Amilcare Porporato, Max Rietkerk and an anonymous reviewer for comments that greatly helped improving the presentation of our results.

\section{References}

[1] I. Rodriguez-Iturbe, A. Porporato, Ecohydrology of water controlled ecosystems, Cambridge University Press, 2005.

[2] I. Noy-Meir, Desert ecosystems: Environment and producers, Annual Review of Ecology and Systematics 4 (1973) 25-51.

[3] I. Rodriguez-Iturbe, Ecohydrology: a hydrologic perspective of climate-soilvegetation dynamics., Water Resources Research 36 (1) (2000) 3-9.

[4] P. D'Odorico, F. Laio, L. Ridolfi, Noise-induced stability in dryland plant ecosystems, Proceedings of the National Academy of Sciences 102 (31) (2005) 10819-10822.

[5] T. M. Scanlon, K. K. Caylor, S. Manfreda, S. A. Levin, I. Rodriguez-Iturbe, Dynamic response of grass cover to rainfall variability: implications for the 
function and persistence of savanna ecosystems, Advances in Water Resources 28 (2005) 291-302.

[6] C. Fernandez-Illescas, I. Rodriguez-Iturbe, Hydrologically driven hierarchical colonization-competition models: the impact of interannual climate fluctuations, Ecological Monographs 73 (2) (2003) 207-222.

[7] P. Chesson, R. L. E. Gebauer, S. Schwinning, N. Huntly, K. Wiegand, M. S. K. Ernest, A. Sher, A. Novoplansky, J. F. Weltzin, Resource pulses, species interactions, and diversity maintenance in arid and semi-arid environments, Oecologia 141 (2004) 236-253.

[8] M. Rietkerk, M. C. Boerlijst, F. V. Langevelde, R. HilleRisLambers, J. V. de Koppel, L. Kumar, H. H. T. Prins, A. M. D. Roos, Self-organization of vegetation in arid ecosystems, American Naturalist 160 (2002) 524-530.

[9] E. Gilad, J. von Hardenberg, A. Provenzale, M. Shachak, E. Meron, Ecosystem engineers: from pattern formation to habitat creation, Physical Review Letters 93 (9) (2004) 098105.

[10] R. Levins, Some demographic and genetic consequences of environmental heterogeneity for biological control, Bulletin of the Entomological Society of America 15 (1969) 237-240.

[11] D. Tilman, Competition and biodiversity in spatially-structured habitats, Ecology 75 (1994) 2-16.

[12] J. T. Lundholm, D. W. Larson, Experimental separation of resource quantity from temporal variability: seedling responses to water pulses, Oecologia 141 (2) (2004) 346-352.

[13] A. A. Sher, D. E. Goldberg, A. Novoplansky, The effect of mean and variance in resource supply on survival of annuals from mediterranean and desert environments, Oecologia 141 (2) (2004) 353-362.

[14] F. Laio, A. Porporato, L. Ridolfi, I. Rodriguez-Iturbe, Plants in water controlled ecosystems: active role in hydrological processes and response to water stress II. Probabilistic soil moisture dynamics, Advances in Water Research 24 (2001) 707-723.

[15] R. G. Allen, L. S. Pereira, M. Smith, D. Raes, J. L. Wright, FAO-56 dual crop coefficient method for estimating evaporation from soil and application extensions, Journal of Irrigation and Drainage Engineering 131 (1) (2005) 213.

[16] A. Porporato, F. Laio, L. Ridolfi, K. K. Caylor, I. Rodriguez-Iturbe, Soil moisture and plant stress dynamics along the kalahari precipitation gradient, Journal of Geophysical Research 108 (2003) 4127-4134.

[17] F. Laio, A. Porporato, C. Fernadez-Illescas, I. Rodriguez-Iturbe, Plants in water controlled ecosystems: active role in hydrological processes and response to water stress IV. Applications to real cases., Advances in Water Research 24 (2001) 745-762. 
[18] A. Porporato, F. Laio, L. Ridolfi, I. Rodriguez-Iturbe, Plants in water controlled ecosystems: active role in hydrological processes and response to water stress III. Vegetation water stress, Advances in Water Research 24 (2001) 725-744.

[19] E.

Daly,

A.

Porporato,

I. Rodriguez-Iturbe, Coupled dynamics of photosynthesis, transpiration, and soil water balance. Part II: stochastic analysis and ecohydrological significance, Journal of Hydrometeorology 5 (2004) 559-566.

[20] K. Hasselmann, Stochastic climate models, part I, theory, Tellus 28 (1976) 473485.

[21] A. Porporato, G. Vico, P. Fay, Superstatistics of hydro-climatic fluctuations and interannual ecosystem productivity, Geophysical Research Letters 33 (2006) L15402.

[22] H. Walter, Natural savannas, in: Ecology of Tropical and Subtropical Vegetation, Oliver and Boyd, Edinburgh, UK, 1971. 